\title{
Growth inhibitory effect of triple anti-tumor gene transfer using Semliki Forest virus vector in glioblastoma cells
}

\author{
JONG-SOO LEE ${ }^{1}$, JUN-HAN LEE ${ }^{2}$, HARYOUNG POO ${ }^{3}$, MI-SUK KIM ${ }^{4}$, \\ SEUNG-HOON LEE ${ }^{4}$, MOON-HEE SUNG ${ }^{1,5}$ and CHUL-JOONG KIM ${ }^{2}$ \\ ${ }^{1}$ BioLeaders Corporation; ${ }^{2}$ Laboratory of Infectious Disease, College of Veterinary Medicine, \\ Chungnam National University; ${ }^{3}$ Functional Proteomics Laboratory, Korea Research Institute of Bioscience and \\ Biotechnology (KRIBB), Daejeon; ${ }^{4}$ Research Institute and Hospital, National Cancer Center, Goyang, Gyeonggi; \\ ${ }^{5}$ Department of Bio \& Nanochemistry, College of Natural Sciences, Kookmin University, Seoul, Korea
}

Received October 13, 2005; Accepted November 25, 2005

\begin{abstract}
The gene delivery of multiple tumor suppressors can provide an efficient tumor therapy in the case of malignant human glioblastomas containing multiple genetic alteration and inactivation. As such, the current study presents a new delivery system that can simultaneously express three antitumor genes using a Semliki Forest virus (SFV) vector in the expectation of combined or synergistic effects of angiogenesis inhibition by angiostatin and apoptosis induction by p53, PTEN and the rSFV particle itself. Recombinant SFV (rSFV) containing three anti-tumor genes (rSFV-Agt/p53/ PTEN) were found to efficiently transduce and express each anti-tumor gene in glioblastoma cells. In addition, rSFV-Agt/ p53/PTEN also resulted in a more effective induction of apoptosis in vitro and inhibition of tumor growth in nude mice when compared with other rSFVs containing only one or two anti-tumor genes. Accordingly, the current results demonstrate that a triple anti-tumor gene transfer using an rSFV vector would be a powerful strategy for regional cancer gene therapy.
\end{abstract}

\section{Introduction}

Malignant glioblastoma is the most common primary tumor occurring in the central nerve system and is characterized by rapid cell proliferation, high invasiveness, genetic alterations and increased vascularity (1-3). A number of new therapies are being developed in glioblastoma treatment, among which is the delivery of DNA encoding tumor suppressor genes to the tumor site by gene delivery systems.

Semliki Forest virus (SFV)-based expression as a useful vector for gene therapy is an ideal delivery system because it

Correspondence to: Dr Chul-Joong Kim, Laboratory of Infectious Disease, College of Veterinary Medicine, Chungnam National University, Daejeon 305-764, Korea

E-mail: cjkim@cnu.ac.kr

Key words: glioblastoma, p53, PTEN, angiostatin, rSFV is suicidal, has a broad host range, replicate in the cytoplasm of host cells, has an inserted gene capacity of at least $5 \mathrm{~kb}$, and can express high levels of recombinant proteins $(4,5)$. This system has already been shown to be effective in recombinant vaccine studies, suggesting that it could be used for in vivo transfer of the prospective genes for inducing antitumor response.

The tumor suppressor gene transferred to a variety of tumor tissues was $p 53$, which plays a central role in regulating cell division and apoptosis $(6,7)$. As such, the introduction of the $p 53$ gene into a $p 53$-deficient tumor or even $p 53$-positive tumor cells inhibited the tumor growth $(8,9)$. Another tumor suppressor gene, PTEN, mapped to $10 \mathrm{q} 23$, has also been observed in a mutated form in primary glioblastoma and other tumor cells (10). Various functional studies of PTEN, including experiments using animals as a model system, have demonstrated that it plays a significant role in inducing G1 cell-cycle arrest and apoptosis, along with regulating cell adhesion, migration, and differentiation $(11,12)$. The introduction of wild-type PTEN into glioblastoma cells containing endogenous mutant alleles has been found to cause growth suppression $(13,14)$.

Angiogenesis is also known to be an essential requirement for progressive growth of brain tumors. Therefore, the inhibition of angiogenesis may represent a potentially powerful approach to tumor therapy. Angiostatin, an internal peptide fragment of plasminogen, has been shown to potently inhibit the migration and proliferation of endothelial cells in vitro and the growth of tumor cells by inducing apoptosis in vivo (15-17). The results of anti-angiogenic gene therapy utilizing an angiostatin gene (agt) have demonstrated this to be an effective anti-tumor method to suppress tumor-induced angiogenesis and tumor growth (18-20). As mentioned above, the gene transfer of p53, PTEN, or agt can be effective in suppressing tumor growth (21). For synergistic anti-tumor effects, the cotransfection of more than one vector containing different anti-tumor genes into tumor cells has also been attempted $(22,23)$. However, the maximal synergy of any anti-tumor effect can not be expected because two or three separate viruses can not be coinfected into identical cells with a high efficiency following in vivo administration. 
A
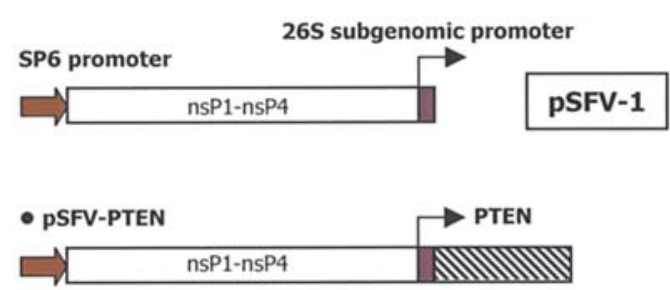

B

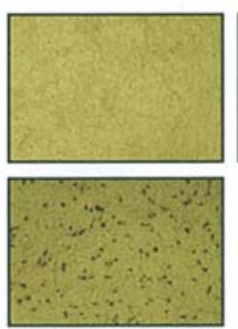

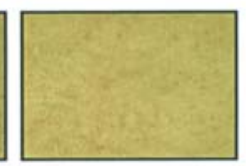
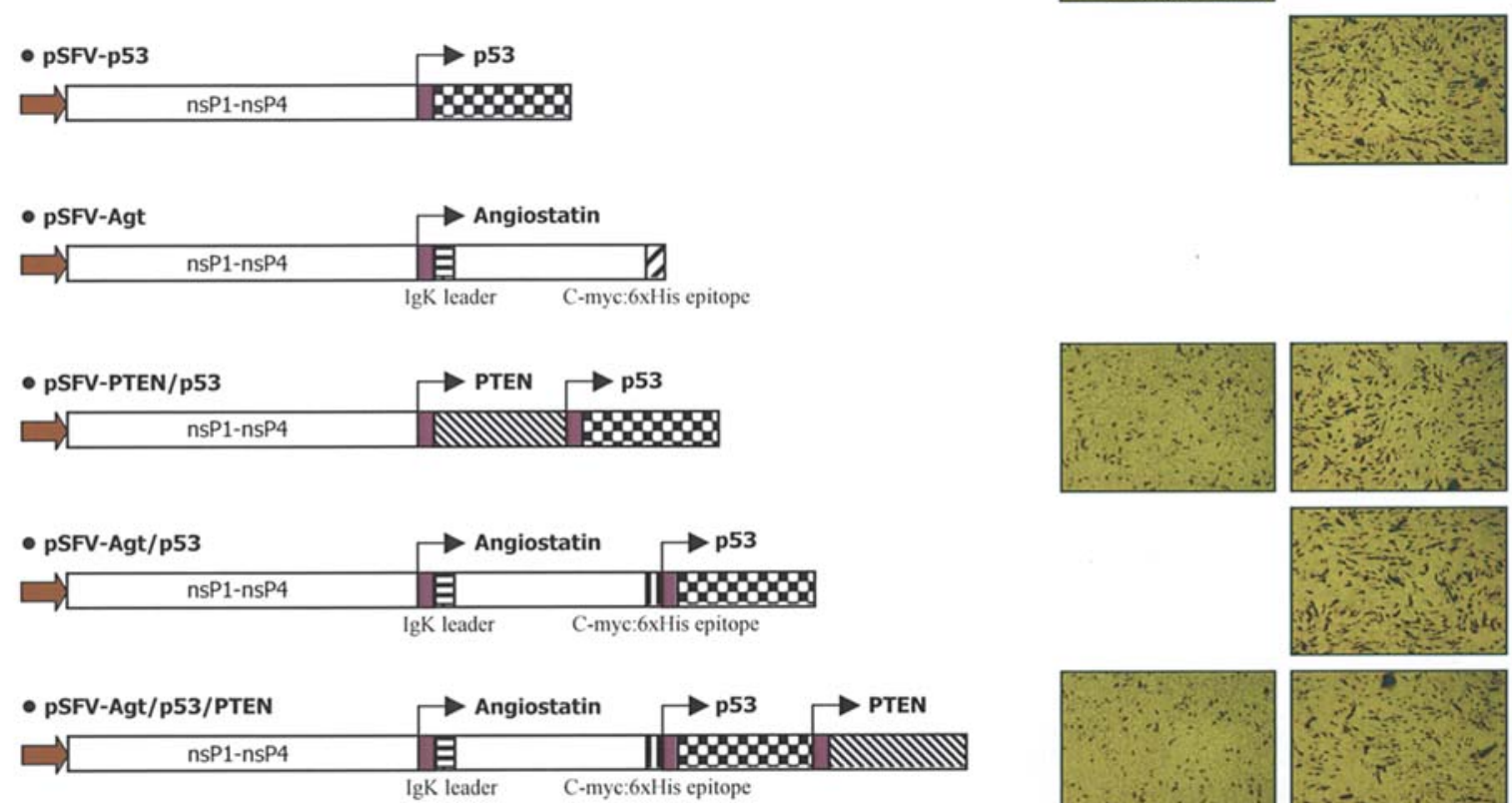

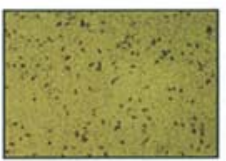

Anti-PTEN
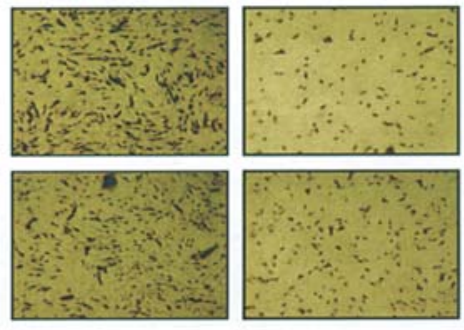

Anti-p53

Anti-c-myc

Figure 1. Schematic diagram of each plasmid (A) and expression of anti-tumor proteins, as shown by immunocytochemistry in BHK-21 cells with anti-PTEN antibody (B, first row), anti-p53 antibody (B, second row) and anti-c-myc antibody (B, third row). The cells were transfected with in vitro transcribed RNAs from the plasmids of pSFV1, pSFV-PTEN, pSFV-p53, pSFV-Agt, pSFV-PTEN/p53, pSFV-Agt/p53, and pSFV-Agt/p53/PTEN, respectively.

In this study, we have used SFV vectors carrying a single anti-tumor gene (pSFV-p53, pSFV-PTEN, and pSFV-Agt) or two or three genes (pSFV-PTEN/p53, pSFV-Agt/p53, and pSFV-Agt/p53/PTEN), all under the control of each subgenomic $26 \mathrm{~S}$ promoter, and we demonstrate the local overexpression of anti-tumor genes in malignant glioblastoma. The rSFV expressing three anti-tumor genes, p53, PTEN, and agt, simultaneously was found to be more efficient in inducing the apoptosis-mediated regression of brain tumors than any of the other rSFVs expressing one or two genes. Consequently, these results indicate that an SFV-based vector offers a very effective system for delivering and expressing functional protein genes concurrently in in vivo gene therapy.

\section{Materials and methods}

Cell culture. The human glioblastoma cell lines, U87MG and U251MG, were prepared and maintained in Dulbecco's modified essential medium (Gibco BRL, Grand Island, NY) supplemented with $10 \%$ fetal bovine serum (Gibco BRL), $2 \mathrm{mM}$ L-glutamine (Sigma, St. Louis, MO), penicillin (0.1 U/ $\mathrm{ml})$, and streptomycin $(0.1 \mu \mathrm{l} / \mathrm{ml})$. Baby hamster kidney cells (BHK-21) were obtained from the Korean Type Culture
Collection (KCTC, Daejon, Korea) and maintained in Dulbecco's minimal Eagle's medium (DMEM) supplemented with $10 \%$ FBS (Gibco BRL).

Construction of recombinant plasmids and rSFVs carrying anti-tumor genes. Human p53, PTEN, and angiostatin cDNAs were obtained from the plasmids, pCEP4-p53, pSecTaq2AAgt, and pcDNA3-PTEN, respectively. The SFV vectors, pSFV1 and pSFV-helper plasmids, were kindly provided by Dr Marc Girard at the Pasteur Institute, France. Each antitumor gene amplified by PCR was inserted independently into the BamHI site of pSFV1 to generate pSFV-p53, pSFVPTEN, and pSFV-Agt. The amplified 1.4-kb angiostatin gene product included a murine Igא chain leader sequence upstream of the angiostatin and c-myc epitope and six tendem histidine residue sequences downstream of angiostatin. The expression cassette for p53, including a subgenomic 26S promoter, was amplified using the pSFV-p53 plasmid as the template. This fragment was then subsequently cloned into pSFV-PTEN and pSFV-Agt to generate pSFV-PTEN/p53 and pSFV-Agt/p53, respectively. The PCR fragment of PTEN, including the $26 \mathrm{~S}$ promoter sequence, was amplified using pSFV-PTEN as the template and then cloned into pSFV-Agt/ 
p53 to generate pSFV-Agt/p53/PTEN (Fig. 1). To generate the rSFVs, in vitro-transcribed RNA from each recombinant plasmid (pSFV-lacZ, pSFV-p53, pSFV-PTEN, pSFV-Agt, pcSFV-Agt/p53, pSFV-PTEN/p53 and pSFV-Agt/p53/PTEN) along with RNA transcribed from the pSFV-helper were cotransfected into the BHK-21 cells by electroporation. The rSFVs were then harvested from the cell culture fluid after $48 \mathrm{~h}$ of incubation by ultracentrifugation at $100,000 \times \mathrm{g}$ for $3 \mathrm{~h}$, resuspended in a TNE buffer $(100 \mathrm{mM} \mathrm{NaCl}, 0.5 \mathrm{mM}$ EDTA, $20 \mathrm{mM}$ Tris- $\mathrm{HCl}, \mathrm{pH} \mathrm{7.4)}$, and stored at $-70^{\circ} \mathrm{C}$ until use. The transfected cells were fixed using $4 \%$ paraformaldehyde and blocked for $30 \mathrm{~min}$ in $1 \%$ gelatin. The blocking solution was then removed and the cells reacted with a primary antibody for $3 \mathrm{~h}$ at room temperature. Next, the cells were rinsed in PBS and reacted with a biotinylated secondary antibody (Vector Laboratories) for $1 \mathrm{~h}$ at room temperature. After reacting with the avidin-biotin reaction solution (Vector Laboratories) for $30 \mathrm{~min}$, a DAB solution containing 3,3'diaminobenzidine tetrahydrochloride and $0.2 \%$ hydrogen peroxide was added. The expression of the anti-tumor genes was finally detected using inverted microscopy.

Infection of rSFVs and Western blot analysis. Each rSFV produced with the helper-packing system was activated using $\alpha$-chymotrypsin (Boehringer Mannheim, Mannheim, Germany). The cells were infected with rSFVs at a multiplicity of infection (MOI) of 10. After $48 \mathrm{~h}$ of incubation, the cells were washed and scraped into PBS, pelleted, and then lysed with $200 \mu \mathrm{l}$ of an RIPA buffer (10 mM Tris- $\mathrm{HCl}, \mathrm{pH} 7.6$, $150 \mathrm{mM} \mathrm{NaCl}, 2 \mathrm{mM}$ EDTA, $1 \%$ Nonidet P-40 and $1 \mu \mathrm{l} / \mathrm{ml}$ PMSF). To detect the secreted angiostatin, the culture supernatant was harvested and concentrated to $1 / 20$ of the original volume. Thereafter, $30 \mu 1$ of each lysate or cell supernatant was resolved using a $12 \%$ discontinuous SDS-PAGE and transferred onto PVDF membranes (Bio-Rad, Hercules, CA). The membranes were probed with an anti-p53, anti-PTEN, or anti-c-myc anti-body, and then the target proteins were detected by chemiluminescence (SuperSignal Substrate, Pierce, Rockford, IL).

Cell viability and tumorigenicity assay. Each line of glioblastoma cells was seeded at $1 \times 10^{4}$ cells/well in 6 -well plates for $20 \mathrm{~h}$ before viral infection. The cells were infected in triplicate with rSFVs produced from each plasmid containing the anti-tumor genes and the $\beta$-galactosidase gene as the control at an MOI of 5 . After $48 \mathrm{~h}$ of incubation, triplicate dishes of each treatment were counted according to the trypan blue exclusion method using a hemocytometer. For tumor formation in the nude mice assay, U251MG cells were plated at $1 \times 10^{6}$ cells/flask. After overnight incubation, the cells were infected with rSFVs, as mentioned above, for $3 \mathrm{~h}$. The infected or mock cells were trypsinized and resuspended in PBS, and then $5 \times 10^{6}$ cells were injected into athymic nu/nu mice (KRIBB). Four weeks after injection, calipers were used to measure the tumor diameters in three dimensions and the tumor volumes were calculated.

Apoptotic cell detection assay. The apoptosis of the infected cells by the rSFVs was assessed using an apoptosis detection system (Promega, Madison, WI) which measured the fragmented DNA of the apoptotic cells using a TUNEL assay. The apoptotic index was analyzed based on the percentage of positive cells using flow cytometry (FACScalibur, BectonDickinson, Franklin Lakes, NJ).

Inhibition of tumor growth in vivo. To determine the inhibition of tumor growth in vivo, nude mice were injected subcutaneously with $1 \times 10^{6} \mathrm{U} 251 \mathrm{MG}$ cells. After developing visible tumors ( $=14$ days post-inoculation), the mice were injected twice at 3 -day intervals with $1 \times 10^{9} \mathrm{rSFV}$ in PBS intratumorally. The tumor volumes were measured using calipers and calculated prior to the virus injection and subsequently at 5-day intervals up to 40 days post-injection.

\section{Results and Discussion}

Construction of SFV vector carrying anti-tumor genes. The alphavirus vector based on SFV has been demonstrated as a favorably comparable gene transfer vector (24). Since this vector is a transient RNA-based expression system, this obviates certain nuclear complications, such as random RNA splicing or host chromosomal integration. The replicon contains plus and minus strand promoters, the genes for the replicase complex, and a cloning site for foreign gene insertion.

The replicon contains the packaging signal and is packaged into virions when helper functions are provided in trans. The defective helper construct only provides genes encoding structural proteins. When these two RNA molecules transcribed in vitro are introduced into host cells, both RNAs are replicated and recombinant SFV (rSFV) particles containing the replicon and a foreign gene are produced. rSFV particles are very stable and can be concentrated to titers exceeding $10^{10}$ infectious units per milliliter. Furthermore, they also have the ability to infect both dividing and non-dividing cells, adsorb rapidly to cells at the injection site, and are capable of one round of multiplication only. In the current study, SFV replicons were constructed carrying either the $p 53, P T E N$, or agt gene under the control of the SFV 26S promoter, resulting in pSFV-p53, pSFV-PTEN, and pSFV-Agt, respectively. To achieve an efficient secretion of the expressed agt, an Igא leader sequence was inserted in front of the agt gene.

In vitro expression of three anti-tumor genes. The tumor suppression activity of the three anti-tumor genes was demonstrated using other viral systems in glioblastoma cells. Previously, the combined gene transfer of two anti-tumor genes based on the co-injection of two separate viral vectors containing different anti-tumor genes was investigated to determine the synergistic effects. Although the co-injection of multiple viral vectors expressing different anti-tumor genes into a tumor was found to be more effective than the injection of a single viral vector including only one anti-tumor gene, it was suggested that treatment with a single vector coexpressing multiple anti-tumor genes might be even more effective. Accordingly, for the simultaneous expression of multiple anti-tumor genes using a single vector, the second and third expression cassettes each containing the $26 \mathrm{~S}$ promoter and functional gene at the end of the first gene were connected, resulting in pSFV-PTEN/p53, pSFV-Agt/p53, and pSFVAgt/p53/PTEN (Fig. 1A). rSFVs containing one, two, or 
A

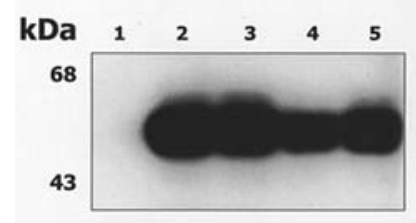

C

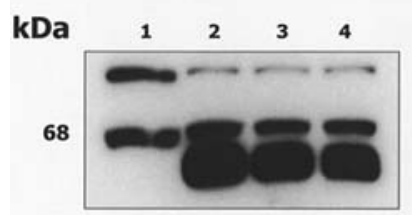

B

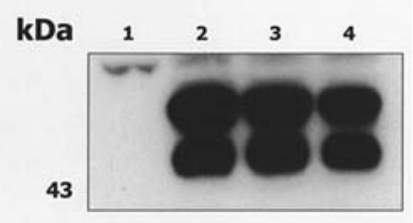

D

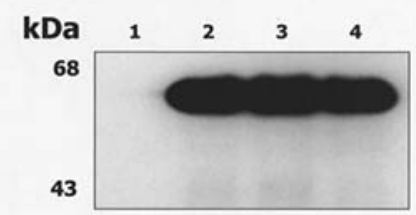

Figure 2. Expression of anti-tumor proteins, p53 (A), PTEN (B), and angiostatin (C) in U251MG cells and secreted angiostatin in supernatant of infected cells (D), as shown by Western blotting. The cells were infected with either rSFV-lacZ (lane 1 of A-D), rSFV-p53 (A, lane 2), rSFV-PTEN (B, lane 2), rSFV-Agt (lane 2 of $\mathrm{C}$ and D), rSFV-PTEN/p53 (lane 3 of A and $\mathrm{B}$ ), rSFV-Agt/p53 (lane 4 of $\mathrm{A}$ and lane 3 of $\mathrm{C}$ and $\mathrm{D}$ ), or rSFVAgt/p53/PTEN (lane 5 of A and lane 4 of B-D).

three different anti-tumor genes were then generated by the cotransfection of BHK-21 cells with RNA transcripts from each expression replicon and helper plasmid. The transient expression of each SFV replicon containing anti-tumor genes in BHK-21 cells was examined 48-h post-transfection by immunocytochemistry using monoclonal antibodies (Fig. 1B). The expression efficiency of each anti-tumor gene, whether cloned alone or with other genes in the SFV vector, seemed to be consistent. In addition, the expression level of each anti-tumor gene was also examined 48-h post-infection with rSFV using Western immunoblotting (Fig. 2). This experiment verified the highly efficient expression of the anti-tumor proteins [p53 (53 kDa), Fig. 2A; PTEN (55 kDa), Fig. 2B; and angiostatin $(64 \mathrm{kDa})$, Fig. $2 \mathrm{C}$ ] in U251MG cells. Angiostatin was also detected in the culture supernatant of the rSFV-Agt infected U251MG cells (Fig. 2D). The expression level of the anti-tumor proteins and infectivity of the rSFV were unaffected by the presence of a second or third gene connected in cis with their own 26S promoter. Accordingly, these results suggest that the SFV polymerase produced a similar amount of subgenomic RNAs to encode the three different anti-tumor proteins, each under the control of a separate $26 \mathrm{~S}$ promoter, thereby enabling the $\mathrm{rSFV}$ to package a replicon RNA containing three different foreign genes with a total size $>4 \mathrm{~kb}$. Consequently, this would appear to be the first report demonstrating the overproduction of three different proteins based on the transfer of a viral vector carrying three different genes into mammalian cells.

In vitro study of synergistic effect of anti-tumor proteins on tumor growth. Glioblastoma cells (U87MG and U251MG) were infected with rSFV-lacZ, rSFV-p53, rSFV-PTEN, rSFV-Agt, rSFV-PTEN/p53, rSFV-Agt/p53, or rSFVAgt/p53/PTEN [multiplicity of infection (MOI) =5]. Because both cell lines express a mutant type of p53 and PTEN, we could examine the unambiguous effect of wild-type p53 and PTEN expressed in infected cells. At $48 \mathrm{~h}$ post infection with rSFV-lacZ, approximately $35 \%$ of the cells had died, which

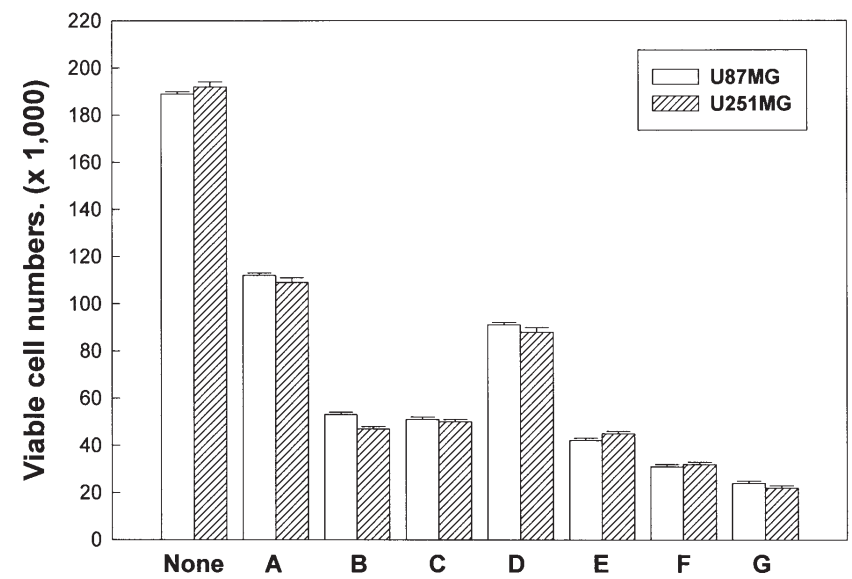

Figure 3. Viable cell count assay of U87MG and U251MG cells infected with rSFVs (none, no infection; A, rSFV-lacZ; B, rSFV-p53; C, rSFVPTEN; D, rSFV-Agt; E, rSFV-Agt/p53; F, rSFV-PTEN/p53; and G, rSFV/Agt/p53/ PTEN). Mean cell numbers $48 \mathrm{~h}$ after infection; bars, SD (three replicates).

coincided with previous results where SFV induced apoptosis in a p53-independent manner (25). Infection with rSFV-p53, rSFV-PTEN, or rSFV-Agt in glioblastoma cells induced approximately $75 \%, 75 \%$, and $50 \%$ cell death, respectively. Approximately $75-80 \%$ cells died after infection with rSFV$\mathrm{PTEN} / \mathrm{p} 53$ or $\mathrm{rSFV}-\mathrm{Agt} / \mathrm{p} 53$. In particular, after infection with rSFV-Agt/p53/PTEN, most cells (>85\%) died (Fig. 3). To check whether the marked increase in the cell death of U251MG, mutated by both the p53 and PTEN genes, was due to apoptosis induced by infection with rSFV, a terminal deoxynucleotidyl transferase-mediated dUTP nick-end labeling (TUNEL) assay was performed and the results were analyzed by flow cytometry.

To assess the apoptosis rate due to the anti-tumor proteins, the infected cells were examined 24-h post-infection. Fig. 4 shows the frequency of apoptosis in U251MG cells infected with rSFVs, as measured by TUNEL assay. After the glioblastoma cells were infected with rSFV-lacZ, an increased number of apoptotic cells were observed compared to the mock control. However, a higher apoptotic frequency was observed in cells infected with rSFVs containing anti-tumor gene(s) than in rSFV-lacZ-infected cells. In particular, cells infected with rSFV-Agt/p53/PTEN showed the highest frequency of apoptosis $(\sim 82 \%)$ compared to any other rSFVinfected cells $(\sim 19-74 \%)$. This result suggests that the three different anti-tumor proteins coexpressed in the glioblastoma cells synergistically induced the apoptosis of U251MG cells.

The cytotoxicity induced by rSFV-lacZ-infected cells suggests that regional rSFV-mediated delivery of anti-tumor proteins may be useful for local tumor control.

In vivo study of synergistic effect of anti-tumor proteins on tumor growth. To check the effect of the rSFVs on tumor growth, U251MG cells infected with rSFVs were injected into nude mice (Table I). For this purpose, U251MG cells were infected with rSFV-lacZ, rSFV-p53, rSFV-PTEN, rSFVAgt, rSFV-Agt/p53, rSFV-PTEN/p53, or rSFV-Agt/p53/PTEN (MOI=10), and then the transduced U251MG cells were injected subcutaneously into nude mice. The tumor size was 
A

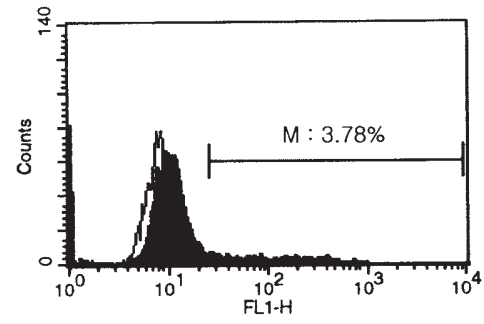

C

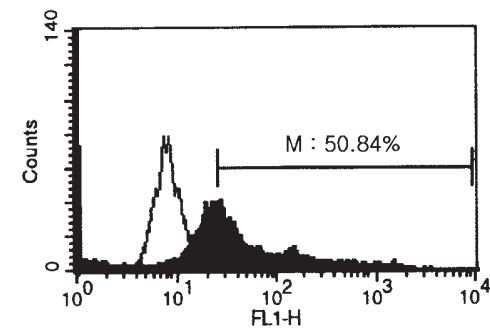

$\mathbf{E}$

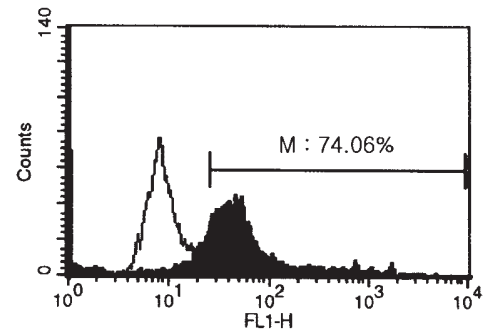

B

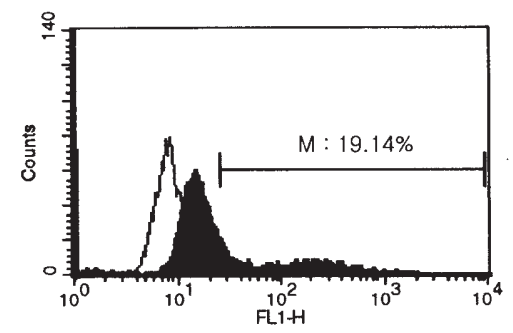

D

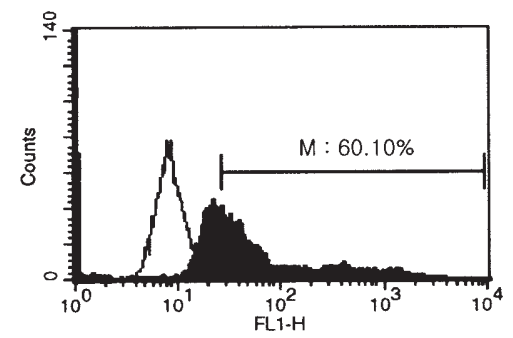

$\mathbf{F}$

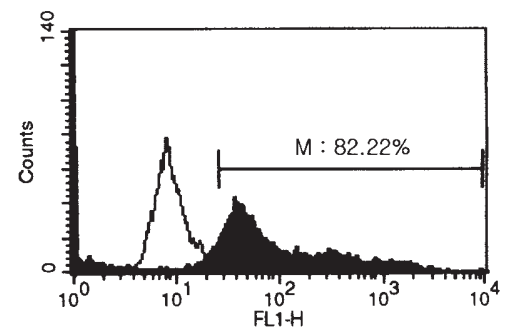

Figure 4. Analysis of apoptosis in U251MG cells based on TUNEL assay. The cells were infected with the rSFVs, then the apoptotic index was analyzed based on the percentage using flow cytometry (A, control; B, rSFV-lacZ; C, rSFV-p53; D, rSFV-Agt/p53; E, rSFV-PTEN/p53; and F, rSFV-Agt/p53/PTEN).

Table I. Tumor growth in nude mice injected with U251MG cells infected ex vivo with rSFVs.

\begin{tabular}{lcc}
\hline Treatment & $\begin{array}{c}\text { No. of mice with } \\
\text { tumors/no. of } \\
\text { mice injected }\end{array}$ & $\begin{array}{c}\text { Mean tumor } \\
\text { volume } \\
\left(\mathrm{mm}^{3} \pm \mathrm{SD}\right)\end{array}$ \\
\hline Mock & $6 / 6$ & $3124 \pm 801$ \\
rSFV-lacZ & $5 / 6$ & $1634 \pm 754$ \\
rSFV-p53 & $5 / 6$ & $726 \pm 194$ \\
rSFV-PTEN & $5 / 6$ & $751 \pm 367$ \\
rSFV-Agt & $6 / 6$ & $692 \pm 259$ \\
rSFV-Agt/p53 & $6 / 6$ & $312 \pm 103$ \\
rSFV-PTEN/p53 & $5 / 6$ & $479 \pm 68$ \\
rSFV-Agt/p53/PTEN & $5 / 6$ & $156 \pm 72$ \\
\hline
\end{tabular}

For the effect of rSFVs on tumor growth ex vivo, the transduction of rSFV-infected U251MG cells was performed. U251MG cells $\left(5 \times 10^{6}\right)$ were infected with $\mathrm{rSFV}$ s $(\mathrm{MOI}=10)$ and then washed $4-5 \mathrm{~h}$ later. These infected cells were introduced subcutaneously into athymic nu/nu mice $(\mathrm{n}=6)$. Tumor volumes were measured after 4 weeks. The average sizes of the tumors and standard deviation are given.

measured 4 weeks post inoculation. The size of the tumors formed by U251MG cells infected with rSFV containing one or two tumor-suppressor genes was $10-25 \%$ of that of the control tumors developed based on the inoculation of uninfected U251MG. The rSFV-Agt/p53/PTEN-infected cells formed tumors that were only $6 \%$ of the size of the control tumors. As such, the growth of the glioblastoma tumor cells was more suppressed by rSFV particles containing a combination of agt, p53, and PTEN than by rSFV particles containing only one or two these genes.

To assess the potential therapeutic efficiency, an in vivo experiment was also conducted using pre-established tumors developed from U251MG glioblastoma in nude nice and the direct injection of rSFVs. Whereas the rSFVs carrying only a single gene exhibited a marginal growth-retarding effect, the coexpression of two genes led to an almost complete inhibition of tumor growth for at least 20 days. In addition, the rSFV carrying the three genes (agt, p53, and PTEN) significantly extended the growth inhibition of tumors to 40 days compared to the other rSFVs carrying one or two genes (Fig. 5). Accordingly, these results provide further support for the use of the SFV vector in cancer gene therapy, particularly for the delivery of three synergistic anti-tumor genes using a single vector. The combined high-level expression of p53, PTEN, and angiostatin substantially increased the regression of the established tumors, compared with the vectors expressing only one or two anti-tumor proteins. The direct intratumoral injection of rSFVs expressing p53, PTEN, and angiostatin simultaneously was demonstrated to be very effective in suppressing tumors by reducing tumor growth or angiogenesis. Therefore, rSFVs expressing p53, PTEN, and angiostatin in combination would appear to represent a highly effective means of in vivo therapy for patients.

With intratumoral injection of rSFVs, the treated mice remained healthy and no lesions were detected in the tissue surrounding the tumor (25). This may be because rSFVs 


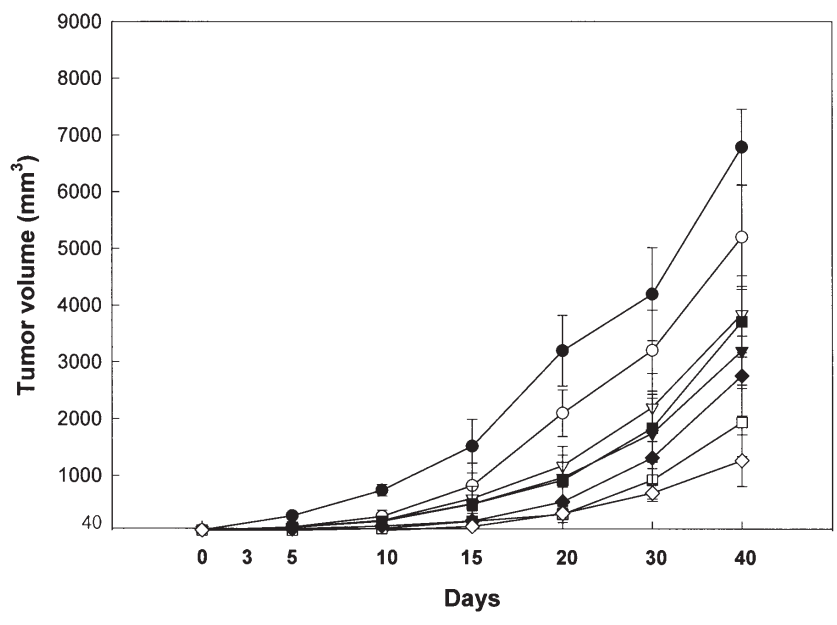

Figure 5. Inhibition of tumor growth following intratumoral injection with rSFVs. U251MG cells $\left(1 \times 10^{6}\right)$ were inoculated into nude mice subcutaneously. At 14 days post inoculation, each $\mathrm{rSFV}\left(1 \times 10^{9}\right)$ was injected into a visible

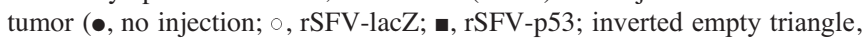
rSFV-PTEN; $\mathbf{\nabla}$, rSFV-Agt; •, rSFV-PTEN/p53; $\square$, rSFV-Agt/p53; and $\diamond$, rSFV-Agt/p53/PTEN). Injection was then repeated 3 days later.

adsorb rapidly to cells at the injection sites and only one round of multiplication is possible. When rSFVs are injected into the brain tumor directly, it is necessary to be cautious because of apoptosis in the normal tissue surrounding the tumor.

As regards the gene transfer of tumor-suppressor genes, several types of viral vectors have been developed. However, most previous experiments have been performed using an adenoviral or retroviral vector system for the delivery of a single tumor-suppressor gene [p53 $(8,9,26,27)$, PTEN $(13,14)$, and pl6INK4 $(23,28)]$ or anti-angiogenic gene $($ agt $)(18-20,29)$. It was reported that the transfer of a recombinant adenovirus encoding a p27-p16 fusion gene (30) and the combined transfer of two recombinant adenovirus encoding the pl6INK4 and p53 genes (23), respectively, led to a more efficient inhibition of tumor growth than the transfer of a recombinant virus carrying only a single tumor-suppressor gene. The current results also suggest that rSFV particles containing three anti-tumor genes, such as p53, PTEN, and agt, would be a convenient and efficient method for delivering three genes into each tumor cell, thereby synergistically inducing the most effective suppression of tumor cell growth.

\section{Acknowledgements}

This work was supported by a National Research Laboratory Program grant (2000-N-NL-01-C-171) from the Korean Ministry of Science and Technology and Biochallenger Program Grant (M1-0310-82-0000) from the Korea Ministry of Commerce, Industry and Energy.

\section{References}

1. James CD and Olson JJ: Molecular genetics and molecular biology advances in brain tumors. Curr Opin Oncol 8: 188-195, 1996.

2. Louis DN and Gusella JF: A tiger behind many doors: multiple genetic pathways to malignant glioma. Trends Genet 11: 412-415, 1995.

3. Westermark B and Nister M: Molecular genetics of human glioma. Curr Opin Oncol 7: 220-225, 1995.
4. Berglund P, Tubulekas I and Liljestrom P: Alphaviruses as vectors for gene delivery. Trends Biotech 14: 130-134, 1995.

5. Frolov I, Hoffman TA, Pragai BM, et al: Alphavirus-based expression vectors: strategies and applications. Proc Natl Acad Sci USA 93: 11371-11377, 1996.

6. Bates S and Vousden KH: p53 in signaling checkpoint arrest or apoptosis. Curr Opin Genet Dev 6: 12-18, 1996.

7. Hartwell LH and Kastan MB: Cell cycle control and cancer. Science 266: 1821-1828, 1994

8. Quazilbash MH, Xiao X, Seth $\mathrm{P}$, et al: Cancer gene therapy using a novel adeno-associated virus vector expressing human wild-type p53. Gene Ther 4: 675-682, 1997.

9. Roth JA, Nguyen D, Lawrence DD, et al: Retrovirus-mediated wild-type p53 gene transfer to tumors of patients with lung cancer. Nat Med 2: 985-991, 1996.

10. Li J, Yen C, Liaw D, et al: PTEN, a putative protein tyrosine phosphatase gene mutated in human brain, breast, and prostate cancer. Science 275: 1943-1947, 1997.

11. Di Cristofano A and Pandolfi PP: The multiple roles of PTEN in tumor suppression. Cell 100: 387-390, 2000.

12. Li DM and Sun H: PTEN/MMAC1/TEP1 suppresses the tumorigenicity and induces $\mathrm{G}_{1}$ cell cycle arrest in human glioblastoma cells. Proc Natl Acad Sci USA 95: 15406-15411, 1998.

13. Cheney IW, Johnson DE, Vaillancourt MT, et al: Suppression of tumorigenicity of glioblastoma cells by adenovirus-mediated MMAC1/PTEN gene transfer. Cancer Res 58: 2331-2334, 1998.

14. Minaguchi T, Mori T, Kanamori Y, et al: Growth suppression of human ovarian cancer cells by adenovirus-mediated transfer of the PTEN gene. Cancer Res 59: 6063-6067, 1999.

15. Griscelli F, Li H, Bennaceur-Griscelli A, et al: Angiostatin gene transfer: Inhibition of tumor growth in vivo by blockage of endothelial cell proliferation associated with a mitosis arrest. Proc Natl Acad Sci USA 95: 6367-6372, 1998.

16. Hanahan D and Folkman J: Patterns and emerging mechanisms of the angiogenic switch during tumorigenesis. Cell 86: 353-364, 1996.

17. Lucas R, Holmgren L, Garcia I, et al: Multiple form of angiostatin induces apoptosis in endothelial cells. Blood 92: 4730-4741, 1998.

18. Griscelli F, Li H, Cheong C, et al: Combined effects of radiotherapy and angiostatin gene therapy in glioma tumor model. Proc Natl Acad Sci USA 97: 6698-6703, 2000.

19. Kirsch M, Strasser J, Allende R, et al: Angiostatin suppresses malignant glioma growth in vivo. Cancer Res 58: 4654-4659, 1998 .

20. Yokoyama Y, Dhanabal M, Griffioen AW, et al: Synergy between angiostatin and endostatin: inhibition of ovarian cancer growth. Cancer Res 60: 2190-2196, 2000.

21. Hinds PW and Weinberg RA: Tumor suppressor genes. Curr Opin Genet Dev 4: 135-141, 1994.

22. Sandig BV, Brand K, Herwig S, et al: p16 and p53 genes transferred with the help of adenovirus to induce apoptotic tumor cell death. Ugeskr Laeger 159: 6825-6830, 1997.

23. Sandig V, Brand K, Herwig S, et al: Adenovirally transferred p16 ${ }^{I N K 4 / C D K N 2}$ and $p 53$ genes cooperate to induce apoptotic tumor cell death. Nat Med 3: 313-319, 1997.

24. Wahlfors JJ, Zullo SA, Loimas S, et al: Evalution of recombinant alphaviruses as vectors in gene therapy. Gene Ther 7: 472-480, 2000.

25. Murphy AM, Morris-Downes MM, Sheahan BJ, et al: Inhibition of human lung carcinoma cell growth by apoptosis induction using Semliki Forest virus recombinant particles. Gene Ther 7: 1477-1482, 2000.

26. Seth P, Brinkmann U, Schwartz GN, et al: Adenovirus-mediated gene transfer to human breast tumor cells: an approach for cancer gene therapy and born marrow purging. Cancer Res 56: 1346-1351, 1996.

27. Swisher SG, Roth JA, Nemunaitis J, et al: Adenovirus-mediated p53 gene transfer in advanced non-small cell lung cancer. J Natl Cancer Inst 91: 763-771, 1999.

28. Craig C, Kim M, Ohri E, et al: Effects of adenovirus-mediated p16 ${ }^{I N K 4 A}$ expression on cell cycle arrest is determined by endogenous p16 and Rb status in human cancer cells. Oncogene 6: 265-272, 1998.

29. Tanaka T, Cao Y, Folkman J and Fine HA: Viral vector-targeted antiangiogenic gene therapy utilizing an angiostatin complementary DNA. Cancer Res 58: 3362-3369, 1998.

30. Patel SD, Tran AC, Ge Y, et al: The p53-independent tumoricidal activity of an adenoviral vector encoding a p27-p16 fusion tumor suppressor gene. Mol Ther 2: 161-169, 2000. 